\title{
Non-linear Characteristic Simulation of Hydraulic Shock Absorbers Considering the Contact of Valves
}

\author{
Liang Liang ${ }^{1}$, Zhang Xiaohong ${ }^{2}$, Peng Manlong ${ }^{1}$, Qin Gang ${ }^{1}$ \\ 1. Huazhong University of Science \&Technology, Wuhan, China \\ 2. Institute of Technology Donghu college, Wuhan University, Wuhan, China \\ e-mail: liang1786@,sohu.com
}

\begin{abstract}
This paper presents a detailed model of a hydraulic shock absorber. The detailed structure includes in the model, such as the deflection of valve which deforms in the oil, the contact between the superposition valves et al. The FEM is used to solve the valve deflection problem. The difference between the FEM result and formula result which is acquired by Mechanical Design Handbook is analyzed. The detailed Mathematical model is simulated by MATLAB and the simulation results fit the experiment data very well. This shows that the model can be used to forecast the performance of hydraulic shock absorber when design.
\end{abstract}

Keywords- Superposition valve, Contact, Non-linear damping characteristic

\section{INTRODUCTION}

Hydraulic shock absorber(HSA) is one of the most complex components in the automobile suspension system, which plays an important role during the vehicle running[1] And it can efficiently prevent the relative motion of the sprung and unsprung mass.

The traditional design methods of HSA are according to experience to determine the design parameter before test modified and the samples with different structural parameters are used to equip to an automobile, then the test evaluations are given by the commissioning members. This method must adjust the inter parameters of HSA and needs trial-manufacture and tests a lot of times. It not only needs a long period and high cost, but also has diffculty to get the optimal HAS characteristics[2]. It is an inevitable trend that the system simulation technology is introduced to forecast and predict the performance of HSA, in order to overcome the disadvantage above mentioned. The basic process of this way is to build its mathematic model and simulate its damping characteristic. Finally, the damping characteristic is used to analyze the vehicle system dynamics in order to evaluate the ride performance and control stability. Consequently, the core problem that system simulation is used to study HSA is to construct the detailed mathematical model. What's more, its damping characteristic can be predicted exactly in design stage[3].

The simulation models of HSA can be divided into two types roughly. The first is the black box model which reflects its external characteristic[4]. It mainly abstracts the absorber as a set of some typical physical components with mechanical properties (e.g. elastic, damping element, friction element etc). Each element composes the damper model through certain constraint relations, and the characteristic parameters of these components are acquired via experimental test. This model is simple and needs less computation, therefore, it is often used in the vehicle system dynamics and vibration simulation. However this model can not reflect the internal structure of the damper in detail, simulate its internal working process precisely and imitate the direct influence in its performance due to the change of its internal construction. The other type is the detailed physical model which is constructed based on the internal structure of the damper[5]. The knowledge about hydromechanics is used to set up the complex coupling dynamic equations that can express the internal structure of HSA. The pressure of each chamber is determined during its working progress. Some scholars utilize the maximum deflection factor and calculation formula in the mechanical design handbook to solve valve deformation during the damper working. However, the mentioned formula could only calculate the maximum deflection of the valve at the external diameter. For the low speed case, calculation results from this method is close to experimental results[6]. Some other scholars employ elasticity theory to study above problem. They formulate a differential equation of the valve deformation and give a very complex general solution[6]. Although the results obtained from HSA model constructed by this way matches with the experimental results very well, this method may not be able to apply to the simulation of damper performance due to its complexity.

\section{MATHEMATICAL MODEL OF HYDRAULIC SHOCK ABSORBER}

The issue about dynamics simulation of HSA is mainly to solve the pressure difference among each chamber in a certain flow rate. The pressure difference among each chamber is closely related to the deflection of the damper valves. The structure sketch of the damper is shown in figure 1 .

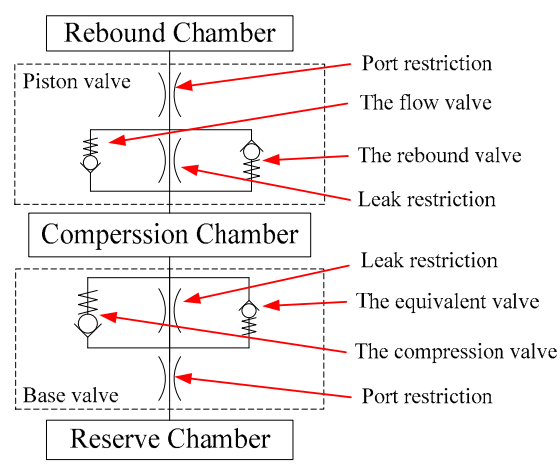

Figure 1. The physical model of shock absorber 
When the piston and piston rod move upward, the oil flows from rebound chamber into compression chamber via the leak restriction and the rebound valve of the piston assembly. Due to the volume of the piston rod, the oil which flows from rebound chamber into compression chamber is not enough to compensate the change of the compression chamber's volume. A part of the oil will flow from the reserve chamber into the compression chamber through the equivalent valve and the leak restriction in the base valve.

As the piston and piston rod move downward, the oil flows from compression chamber into rebound chamber via the leak restriction and the flow valve of the piston assembly. Due to the volume of the piston rod, the oil which flows from compression chamber into rebound chamber is bigger than the change of the compression chamber's volume. A part of the oil will flow from compression chamber into reserve chamber through the compression valve and the leak restriction in the base valve.

During the HSA working, there is pressure difference when the oil flows through these valve and orifice. According to fluid mechanics, the relationship between the flow and the pressure of viscous liquid which flows the orifice is expressed as follows:

$$
Q=C_{d} A \sqrt{\frac{2 \Delta p}{\rho}}
$$

Where $\mathrm{Q}$ denotes oil flow, Cd and A are flow coefficient and flow area, respectively. $\Delta p$ is the pressure difference between the upside and downside of orifice and $\rho$ is oil density.

From the above formula, if circulation area of the oil is obtained, the pressure difference in every chamber is acquired. Then according to the initial conditions of HSA, accurate oil pressure of every chamber is received. At last the damping force is acquired using the following formula as the HSA works.

$$
F=A_{p} P_{2}-\left(A_{p}-A_{r}\right) P_{1}-F_{f} \operatorname{sign}(v)
$$

Where $\mathrm{F}$ denotes the damping force, Ap and Ar are area of the piston and the piston rod, respectively. Ff is the friction between piston and cylinder.

\section{Calculating Large Deflection OF SUPERPOSITION VALVES CONSIDERING CONTACT}

The deformation of the valve has a great influence to the characteristic curve of HSA $[7,8,9]$. It is very important to calculate its deformation accurately, especially for simulation the characteristic of a whole HSA. Comparing with the single valve, the superposition valve can improve the stress state, reduce the maximum bending stress of the valve and make the valve thickness standardized and serialized.

Figure 2 shows the piston valve assembly of a real HAS and its finite element model. The rebound valve is composed of two valves which is equal in thickness. When working, the valve will deform under the action of the hydraulic and a orifice is formed.
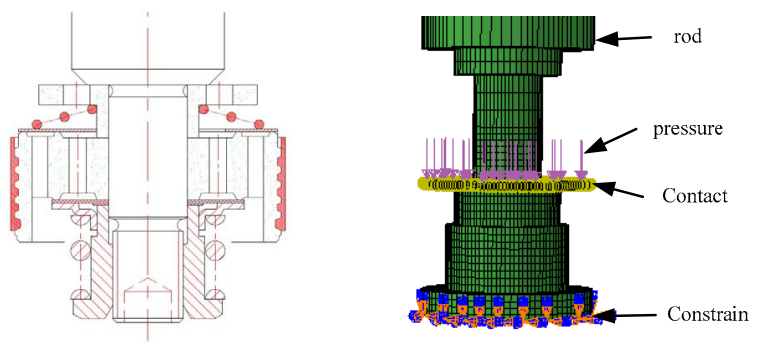

Figure 2. Schematic diagram and finite element model of piston valve

FEM is introduced for calculating the deformation of the Superposition valve. Firstly, the 3D geometry of piston valve assembly is established by UG. Then it is imported into Hypermesh for meshing. Hexahedral elements are used to mesh the piston valve seat, piston rod and shell elements for the superposition valves to ensure the model accuracy and convergence. Finally, the FEM model is solved by Abaqus after defining the boundary condition. A finite sliding contact is defined between the valves.

In the finite element model, the outer radius of valve $a=16 \mathrm{~mm}$, its inner radius $b=8 \mathrm{~mm}$, its thickness $\mathrm{h}=0.3 \mathrm{~mm}$, Young's modulus E $=206 \mathrm{GPa}$, Poisson's ratio $\mu=0.3$, density $\rho=7800 \mathrm{~kg} / \mathrm{m} 3$, the pressure $q=0.2 \mathrm{MPa}$. Because of the surface treatment on the valve, the friction between valves is ignored in FEA model. Figure 4 shows the two results. One is calculated by the Abaqus software, the other is obtained by the Formula in Mechanical Design Handbook.

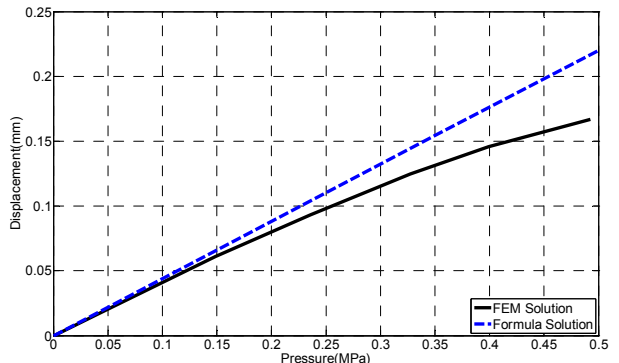

Figure 3. the valve deflection got by FEM and classical Formula

In figure 3 , two results are extremely closed, when the deformation of the valve is very small. As the deformation increasing, however, the deviation becomes more and more obvious. The reason for this error occurred is that the longitudinal displacement in the middle face was so great that it can not be ignored when the deformation of the valve increased.

IV. VALIDATING THE DAMPING CHARACTERISTICS OF SiMUlation MOdEL FOR HYDRAULIC SHOCK ABSORBER

The simulation results of the damping characteristics for the HSA must be validated by experiment. In general, the simulation model and the test sample must be at the same input and the same operating environment so that the comparison is credible and the mathematical model is accurate in some extent. Actually, the damping force of HSA which is installed on the bench test is obtained through a 
typical test condition. At the same time, this test condition is exerted at the simulation model as its input conditions. In other words, the simulation model and the test sample have the same boundary conditions. In this way, the results of these two models can be compared.

The damping characteristic of HSA is tested by dampers test bench. In this paper, the test bed-ElectroForce 3500 is developed by BOSE company which is founded in United States. Figue 4 shows the test bench and its working principle. It is composed of three test systems: Software control system, Data Acquisition System and Dynamic Test System.
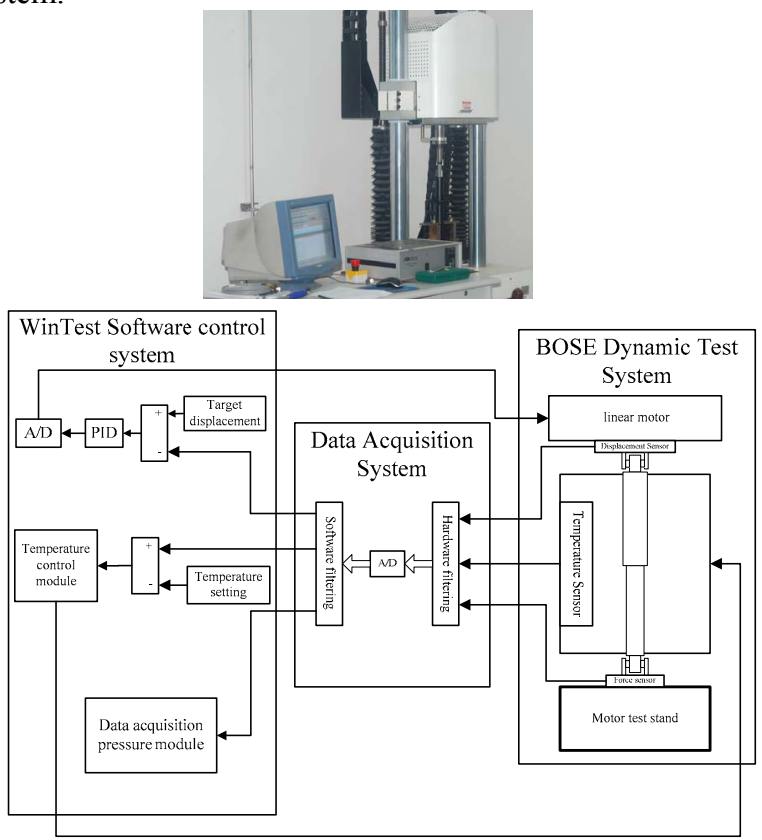

Figure 4. The test Bench and principle of the ElectroForce 3500

When the linear motor which is installed on the top of the test bed works in some frequency, it can make the test sample reciprocating motion in a certain frequency. At the same time, Data Acquisition System of the test bed records and outputs the damping force and displacement of the HSA.

According to national standards, a $50 \mathrm{~mm}$ sinusoidal excitation is applied to HSA. Its damping curve is obtained at the maximum excitation velocity of $0.1 \mathrm{~m} / \mathrm{s}, 0.3 \mathrm{~m} / \mathrm{s}, 0.52$ $\mathrm{m} / \mathrm{s}$ which is acquired through changing the excitation frequency. At the same time, the detailed mathematical model is applied this excitation and solved using MATLAB software. Figure 5 shows the difference between the simulation results and testing results. The simulation model and experimental results is almost unanimous, when the HSA is endured at a lower frequency. As the excitation frequency raised, a little difference appeared, however, the deviation is so small that it can be ignored. From the figure, the precision is above $90 \%$. Therefore, the model can be used to forecast the performance of the HSA when design.

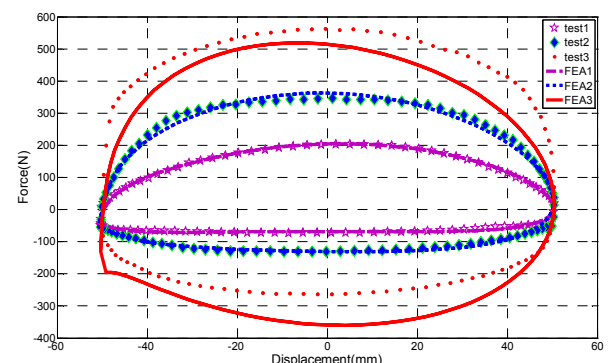

Figure 5. Simulation results VS Testing results

\section{CONCLUSION}

This paper shows a detailed mathematical model of hydraulic shock absorber. The deflection of Superposition valve which is buckled in the oil is considered in this model. FEM was used to solve the valve deflection problems. The difference between the FEM result and Formula result which is obtained by Mechanical Design Handbook was analyzed. Finally, the FEM result is used in the detailed mathematical model of hydraulic shock absorber. At last, it was solved by the MATLAB software and validated using test data. The results show that the precision is above $90 \%$, which can fulfill the engineering requirement.

\section{ACKNOWLEDGMENT}

The authors gratefully acknowledge the financial support by the National High-Tech R\&D Program, China (No. 2009AA044501)

\section{REFERENCES}

[1] Yu De-fu. Chen Qingdong, Fang Dayu. Design Study of Smoothnessto-Safety Ratio in Suspension Shock Absorber outer Characteristic. Vehicle \& Power Technology 87(3): pp.12-17, 2002(in Chinese)

[2] Li Shi-min, Lv Zhen-hua. Technology Development of Cylindrical Fluid Resistance Shock Absorber for Motor Vehicle. Automobile Technology. 8: pp.10-16, 2001(in Chinese)

[3] Zhou Changcheng. Math model for throttle slice thickness analytical design of telescopic shock absorber. International Journal of Vehicle Systems Modeling and Testing, 4(3): pp.133-149, 2009

[4] Duym S, Stiens R, Reybrouck K. Evaluation of shock absorber models. Vehicle System Dynamics, 27: pp.109-127, 1997

[5] Duym S. Simulation tools, modeling and identification, for an automotive shock absorber in the context of vehicle dynamics. Vehicle System Dynamics, 33: pp.261-285, 2000

[6] Basso R. Design of a single-tube shock absorber with a preestablished characteristic diagram. International Journal of Heavy Vehicle System, 17(2): pp.179-195, 2010

[7] Zhou Changcheng. Modeling and simulation of throttle slice stress of telescopic shock absorber. International Journal of Modeling, Identification and Control, 7(1): pp.3-7, 2009

[8] Boggs C, Southward S. Efficient empirical modeling of a highperformance shock absorber for vehicle dynamics studies. Vehicle System Dynamics, 48(4): PP.481-505, 2010

[9] M. Weigel, W. Mack, A. Riepl. Nonparametric Shock Absorber Modelling Based on Standard Test Data. Vehicle System Dynamics, 38(6): PP.415-432, 2002 